\title{
Does the nonlinear Schrödinger equation correctly describe beam propagation?
}

\author{
Nail Akhmediev and Adrian Ankiewicz \\ Optical Sciences Center, Institute of Advanced Studies, Australian National University, Canberra, ACT 2601, Australia
}

Jose Maria Soto-Crespo

Instituto de Optica, Consejo Superior de Investigaciones Científicas, Serrano 121, 28006 Madrid, Spain

Received November 17, 1992

\begin{abstract}
The parabolic equation (nonlinear Schrödinger equation) that appears in problems of stationary nonlinear beam propagation (self-focusing) is reconsidered. It is shown that an additional term, which involves changes of the propagation constant along the propagation direction, should be taken into account. The physical consequences of this departure from the standard approximation, which uses the parabolic equation, are discussed. A numerical simulation showing the difference between the new approach and the standard nonlinear Schrödinger equation is given as an example.
\end{abstract}

The effects of self-trapping and self-focusing of light beams in nonlinear media were predicted in the early 1960 's. ${ }^{1-3}$ The evolution of light beams in self-focusing media has been described by the parabolic equation [the nonlinear Schrödinger equation (NLSE)] since the first studies devoted to this question. 4 This equation takes diffraction and nonlinearity into account in a simple way and describes the field evolution with high accuracy, unless time dependence and dispersion are involved. ${ }^{6}$ Thus, the parabolic equation is a convenient approximation; it provides the possibility of using a powerful tool such as the inverse-scattering method ${ }^{7}$ for its investigation. In fact, a variety of exact solutions can be obtained for the one-dimensional NLSE by using even simpler approaches. ${ }^{8}$ This equation, with variable coefficients adjusted for a layered medium, has been used widely for describing nonlinear wave propagation in optical waveguides and interfaces..$^{9-14}$ It is also important to note that many fast and convenient calculation methods have been developed ${ }^{15}$ for numerical simulations of solutions of the parabolic equation.

Unfortunately, this equation has limitations that have not been discussed before and that are connected with the approximation of slowly varying amplitude. As can be seen in what follows, some physical implications of this equation can even be misleading and turn out to be in contradiction with the general theory of wave propagation. The question arises: Is approximation by the parabolic equation good enough to describe nonlinear guidedwave phenomena in their full complexity?

In this Letter we show that the parabolic equation is a good approximation, unless stationary (in the longitudinal direction) solutions (like self-trapping ${ }^{2}$ and stationary nonlinear guided waves ${ }^{9-14}$ ) or solutions close to them are considered. If the beam has any longitudinal variation during propagation, as happens in most cases of interest, then the equation should be completed by an additional term describing longitudinal field oscillations. This additional term allows us to present a clear physical interpretation of the conserved quantities involved.

For simplicity we start from the scalar wave equation for a three-dimensional field $E(x, y, z)$ in a medium $^{16}$ :

$$
E_{x x}+E_{y y}+E_{z z}-\frac{\epsilon(x,|E|)}{c^{2}} \frac{\partial^{2} E}{\partial t^{2}}=0
$$

where $E$ is a scalar ( $y$-component) optical field and $\epsilon(x,|E|)$ is the intensity-dependent dielectric permittivity:

$$
\epsilon(x,|E|)=\epsilon_{1}(x)+\alpha(x)|E|^{2} .
$$

Here $\epsilon_{1}(x)$ is the linear part of the transverse profile of the dielectric permittivity of the layered medium and $\alpha(x)$ is the transverse profile of the nonlinear susceptibility. In order to include layered media in this analysis we allow $\epsilon$ and $\alpha$ to depend on $x$. This is relevant to problems of nonlinear guided waves. ${ }^{9-14}$ In the particular case of a homogeneous medium, they are constants. The averaged dielectric permittivity does not depend on time. The field is assumed to be stationary (in time) and monochromatic:

$$
E=\psi(x, y, z) \exp (-i \omega t) .
$$

For convenience, we normalize the coordinates $x, y$, and $z$ by using the free-space wave number $k=\omega / c$ :

$$
\psi_{z z}+\psi_{x x}+\psi_{y y}+\epsilon_{1}(x) \psi+\alpha(x)|\psi|^{2} \psi=0 .
$$

We seek a solution of Eq. (2) of the form

$$
\psi(x, z)=B(x, y, z) \exp [i \varphi(z)] .
$$

We assume that the amplitude function $B(x, y, z)$ is slowly varying and that all fast oscillations are included by means of the phase function $\varphi(z)$. Usually, 
in the approximation of slowly varying amplitude, we set $\varphi(z)=\beta z+\varphi_{0}$, with $\beta=$ constant. In this case, fast oscillations are still implicitly included in the function $B(x, y, z)$. However, the second derivative of this function is then not small and cannot be dropped completely.

There can be some degree of arbitrariness in separating the fast oscillatory part from the function $B(x, y, z)$ if the beam divides into two or more beams during propagation. In this instance it is more convenient in numerical simulations to keep $\beta$ constant, as is usually done. The correction to the real value of $\beta$ (separate for each beam) can then be extracted from the results by calculating the period of longitudinal oscillations of $B(x, y, z)$ at the center of each beam. On the other hand, the correct initial separation produces physically important consequences as well as crucial differences in numerical results, as we show below.

We assume that we have only one beam as a solution of Eq. (1), so that the function $|B(x, y, z)|$ has only one maximum at any fixed $z$ and that it approaches zero at $x=\infty$. Substituting Eq. (3) into Eq. (2), we obtain

$$
\begin{aligned}
B_{z z}+B_{x x}+B_{y y} & +2 i \varphi_{z} B_{z}+i \varphi_{z z} B \\
& -\varphi_{z}{ }^{2} B+\epsilon_{1}(x) B+\alpha(x)|B|^{2} B=0 .
\end{aligned}
$$

The term $B_{z z}$ can be omitted from Eq. (4), as is usual in the slowly varying amplitude approximation. However, this can only be done if $B(x, y, z)$ does not include any fast field oscillations in the $z$ direction. With our definition, the rapidly oscillating part of the solution is included by means of the function $\varphi(z)$ in such a way that the function $B(x, y, z)$ maintains constant phase at the center of the beam where $|B(x, y, z)|$ has its maximum. If the beam center is located at $(x, y)=(0,0)$ then we can define the function $\varphi(z)$ in such a way that

$$
\arg [B(x=0, y=0, z)]=\text { constant }
$$

where $B(0,0, z)$ has been written as $|B(0,0, z)| \times$ $\exp [i \arg B(0,0, z)]$. This in turn means that the ratio of the imaginary to the real part of $B(0,0, z)$, i.e., $\operatorname{Im}[B(0,0, z)] / \operatorname{Re}[B(0,0, z)]$, remains constant. Equation (5) defines the functions $\varphi(z)$ and $B(x, y, z)$ uniquely.

Let us now define the function $\tilde{\beta}$ as

$$
\tilde{\beta}(z)=\frac{\mathrm{d} \varphi}{\mathrm{d} z} .
$$

This is the instantaneous propagation constant at a given cross section $z$. This function is also defined in a unique way. Now we can write Eq. (4) in the form

$$
\begin{aligned}
2 i \tilde{\beta} B_{z}+i \tilde{\beta}_{z} B+B_{x x} & +B_{y y}-\tilde{\beta}^{2} B \\
& +\epsilon_{1}(x) B+\alpha(x)|B|^{2} B=0 .
\end{aligned}
$$

In the case of constant $\tilde{\beta}$, this equation obviously coincides with the standard parabolic equation for studying the propagation of nonlinear guided waves. The second term in Eq. (7), which can be of the same order as the first one, makes it different from the usual parabolic equation.

Now let us turn to physical differences that appear when we take this term into account. Consider, for example, the invariants of Eq. (7). By multiplying Eq. (7) by $B^{*}$, taking the complex conjugate of this expression, and subtracting and integrating, we obtain

$$
\frac{\mathrm{d}}{\mathrm{d} z}(\tilde{\beta} I)=0,
$$

where $I$ is the energy invariant for the standard parabolic equation [i.e., Eq. (7) without the second term]:

$$
I=\int_{-\infty}^{\infty}|B(x, y, z)|^{2} \mathrm{~d} x \mathrm{~d} y .
$$

We can see now that the product $\tilde{\beta} I$, rather than just $I$, is the conserved quantity during propagation:

$$
\tilde{\beta}(z) I(z)=\text { constant. }
$$

This product $S_{z}=\tilde{\beta} I$ is proportional to the integrated energy flow ${ }^{16}$ in the $z$ direction, i.e., to the $z$ component of Poynting vector $\mathbf{S}=\mathbf{E} \times \mathbf{H}$ integrated over the cross section. It can also be called power ${ }^{17}$ or power flow ${ }^{18}$ crossing a given surface $z=$ constant. This is the result that we would expect physically, because in the general theory of wave propagation, the energy flow is the quantity that has to be conserved in media without gain or loss. However, in the standard approximation by the parabolic equation, this conservation law is incomplete, and only the energy integral $I$ is conserved, as rapid oscillations are neglected when the term $B_{z z}$ is dropped. Of course, in the case of constant $\beta$, the energy invariant $I$ itself is conserved. This can happen only for stationary solutions of Eq. (2). But in that case it is a trivial result, as the function $B(x, y)$ itself then does not depend on $z$, and so neither will any integral of it. For any other solutions, we have to take into account rapid field oscillations along the $z$ axis.

Let us now consider the second quantity conserved for the parabolic equation, namely the Hamiltonian. For simplicity we restrict ourselves to the case of a two-dimensional field $B(x, z)$. It is now proportional to the $y$ component of the electric field. By multiplying Eq. (7) by $\mathrm{d} B^{*} / \mathrm{d} z$, taking the complex conjugate of this expression, and adding and integrating over $x$, we obtain

$$
\begin{aligned}
& i \int_{-\infty}^{\infty} \mathrm{d} x \tilde{\beta}_{z}\left(B_{z} B^{*}-B_{z}{ }^{*} B\right)-2 \int_{-\infty}^{\infty} \mathrm{d} x \tilde{\beta} \tilde{\beta}_{z}|B|^{2} \\
& +\frac{\mathrm{d}}{\mathrm{d} z} \int_{-\infty}^{\infty} \mathrm{d} x\left[\left|B_{x}\right|^{2}+\left(\tilde{\beta}^{2}-\epsilon_{1}\right)|B|^{2}-\frac{\alpha}{2}|B|^{4}\right]=0 .
\end{aligned}
$$

The sum of integrals in Eq. (11) is proportional to the energy density of the optical field integrated over $x$ per unit $z$. The first two integrals are the part of the energy density connected with the $x$ component of the magnetic field $\left(H_{x}^{2}\right)$. These two integrals become 


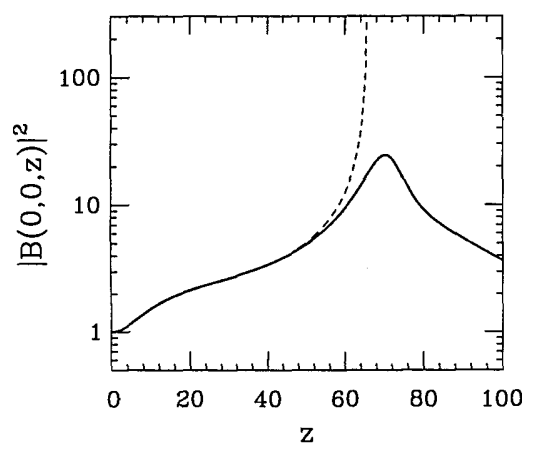

Fig. 1. On-axis intensity versus normalized axial distance $z$ for the Gaussian initial condition $(z=0)$ of Eq. (12) found by using the standard NLSE (dashed curve) and by using Eq. (7) (solid curve). Parameters chosen for these simulations are $\epsilon_{1}=24.50015104$, $\beta(0)=5.0$, and $\alpha=1.0$.

zero when $\beta$ is constant in the standard parabolic approximation. Then the third integral is conserved along $z$. It still can be considered as the energy density per unit $z$. So, in the absence of currents, the integrated energy density is conserved along $z$. If $\tilde{\beta}_{z}$ is nonzero, then the whole Eq. (11) cannot be represented in the form of a conservation law. The consequence is that the Hamiltonian [the third integral in Eq. (11)] is no longer conserved.

In order to show that the new equation gives results that are different from the standard NLSE but are consistent with the solutions of the wave equation, we present here one example of a numerical simulation to compare the two approaches. For this short communication we choose the most striking example where the standard NLSE fails to give a correct result, ${ }^{19}$ namely catastrophic beam collapse in the two-transverse-dimensional NLSE. We have developed a modification of the CrankNicholson scheme that maintains a constant phase of $B(x, y, z)$ at $(x, y)=(0,0)$ to simulate the solutions of Eq. (7). We have verified during simulations that the invariant given by Eq. (10) is conserved to high accuracy for a variety of initial conditions. Figure 1 shows the on-axis intensity of the cylindrically symmetric beam that initially $(z=0)$ has a Gaussian shape:

$$
B(x, y, z=0)=\exp \left(\frac{x^{2}+y^{2}}{8}\right)
$$

This initial condition has a power flow that is above the critical value and thus results in collapse when the standard NLSE is used. The parameters of the simulation are given in the figure caption. It is seen from this figure that the standard NLSE leads to collapse and that the on-axis field goes to infinity at finite $z(\approx 62.5)$. The solution of Eq. (7) deviates from it for on-axis intensities $|B(0,0, z)|^{2}$, which are between approximately 5 and 10 , and then returns to a more smooth beam propagation beyond the point of collapse. This result is in qualitative agreement with the numerical results of Refs. 19 and 20, where the authors use nonparaxial algorithms for simulation of the solution of the wave Eq. (2) to describe beam self-focusing. Thus the NLSE leads to an erroneous physical conclusion.

In summary, we have shown that the standard approximation, which uses the parabolic equation and which is employed widely in the analysis of nonlinear self-focusing and self-guiding, should be completed with an additional term that takes into account the variation of the propagation constant along the propagation direction.

Nail Akhmediev thanks Ewan Wright for fruitful discussions at the First Nonlinear Guided-WaveTheory Workshop, Orlando, Florida, April 1992, and during the Integrated Photonics Research Conference in New Orleans, Louisiana, April 13-16, 1992.

\section{References}

1. G. A. Askar'yan, Zh. Eksp. Teor. Fiz. 42, 1567 (1962) [Sov. Phys. JETP 15, 1088 (1962)].

2. R. Y. Chia, E. Garmire, and C. H. Townes, Phys. Rev. Lett. 13, 479 (1964).

3. V. I. Talanov, Izv. Vyssh. Uchebn. Zaved. Radiofiz. 7, 564 (1964) [Radiophysics 7, 254 (1964)].

4. P. L. Kelley, Phys. Rev. Lett. 15, 1005 (1965).

5. V. E. Zakharov, V. V. Sobolev, and V. S. Synakh, Zh. Eksp. Teor. Fiz. 60, 136 (1971) [Sov. Phys. JETP 33, 77 (1971)].

6. V. E. Zakharov and A. M. Rubenchik, Zh. Eksp. Teor. Fiz. 65, 997 (1973) [Sov. Phys. JETP 38, 494 (1973)].

7. V. E. Zakharov and A. B. Shabat, Zh. Eksp. Teor. Fiz. 61, 118 (1971) [Sov. Phys. JETP 34, 62 (1972)].

8. N. N. Akhmediev, V. M. Eleonskii, and N. E. Kulagin, Theor. Math. Phys. 72, 809 (1987) [Teor. Mat. Fiz (USSR) 72, 183 (1987)].

9. C. T. Seaton, G. I. Stegeman, and H. G. Winful, Opt. Eng. 24, 593 (1985).

10. G. I. Stegeman, E. M. Wright, N. Finlanson, R. Zanoni, and C. T. Seaton, IEEE J. Lightwave Technol. 6, 953 (1988).

11. D. Mihalache, M. Bertolotti, and C. Sibilia, in Progress in Optics, E. Wolf, ed. (North-Holland, Amsterdam, 1989), Vol. XXVII, pp. 229-313.

12. N. N. Akhmediev, in Modern Problems in Condensed Matter Sciences, H.-E. Ponath and G. I. Stegeman, eds. (North-Holland, Amsterdam, 1991), Vol. XXIX, pp. $289-321$.

13. A. D. Boardman, P. Egan, T. Twardowski, and M. Wilkins, in Nonlinear Waves in Solid-State Physics, A. D. Boardman, M. Bertolotti, and T. Twardowski, eds. (Plenum, New York, 1990), pp. 1-50.

14. S. Maneuf, R. Desailly, and C. Froehly, Opt. Commun. 65, 193 (1988); J. S. Aitchinson, A. M. Weiner, Y. Silberberg, M. K. Oliver, J. L. Jackel, D. E. Leaird, E. M. Vogel, and P. W. E. Smith, Opt. Lett. 15, 471 (1990).

15. T. R. Taha and M. J. Ablowitz, J. Comput. Phys. 55, 203 (1984).

16. M. Born and E. Wolf, Principles of Optics, 6th ed. (Pergamon, Oxford, 1980).

17. A. W. Snyder and J. D. Love, Optical Waveguide Theory (Chapman and Hall, London, 1983).

18. $\mathrm{H}$. A. Haus, Waves and Fields in Optoelectronics (Prentice-Hall, Englewood Cliffs, N.J., 1984).

19. M. D. Feit and J. A. Fleck, J. Opt. Soc. Am. B 5, 633 (1988).

20. J. T. Manassah and B. Gross, Opt. Lett. 17, 976 (1992). 